Pure and Applied Mathematics Quarterly

Volume 4, Number 3

(Special Issue: In honor of

Fedor Bogomolov, Part 2 of 2 )

$743-766,2008$

\title{
Approximation at Places of Bad Reduction for Rationally Connected Varieties
}

\author{
Brendan Hassett and Yuri Tschinkel
}

\begin{abstract}
This paper addresses weak approximation for rationally connected varieties defined over the function field of a curve, especially at places of bad reduction. Our approach entails analyzing the rational connectivity of the smooth locus of singular reductions of the variety. As an application, we prove weak approximation for cubic surfaces and Fano hypersurfaces of dimension at least three, with square-free discriminant.
\end{abstract}

\section{Contents}

1. Introduction 744

2. Notions of approximation $\quad 747$

3. Curves, combs, and deformations 749

4. Strong rational connectivity 750

5. Cubic surfaces 757

6. Higher-dimensional Fano hypersurfaces 761

References $\quad 764$

Received April 22, 2006. 


\section{INTRODUCTION}

In number theory, many results and techniques rely on approximating adelic points by rational points. In this paper, we study geometric versions of these notions for rationally connected varieties over the function field of a curve. In this context, rational points correspond to sections of rationally-connected fibrations over the curve. We are looking for sections with prescribed jet data in finitely many fibers.

Let $k$ be an algebraically closed field of characteristic zero, $B$ a smooth curve over $k$ with function field $F=k(B)$. Let $\bar{B}$ be the smooth projective model of $F$ and put $S:=\bar{B} \backslash B$.

Theorem 1. Let $X$ be a smooth proper rationally connected variety over $F$, and $\pi: \mathcal{X} \rightarrow B$ a proper model of $X$. (A model of $X$ is an algebraic space flat over $B$ with generic fiber $X$.) Let $\mathcal{X}^{\text {sm }}$ be the locus where $\pi$ is smooth and $\mathcal{X}^{\bullet} \subset \mathcal{X}^{\text {sm }}$ be an open subset such that

(1) there exists a section $s: B \rightarrow \mathcal{X}^{\bullet}$;

(2) for each $b \in B$ and $x \in \mathcal{X}_{b}^{\bullet}$, there exists a rational curve $f: \mathbb{P}^{1} \rightarrow \mathcal{X}_{b}^{\bullet}$ containing $x$ and the generic point of $\mathcal{X}_{b}^{\bullet}$.

Then sections of $\mathcal{X}^{\bullet} \rightarrow B$ satisfy approximation away from $S$ (see Section 2).

We shall actually prove a stronger result, Theorem 15, that is applicable in positive characteristic. Rationally-connected fibrations over curves have sections by [8]. The existence of a section through a finite set of prescribed points is addressed in [16] 2.13 and [15] IV.6.10.1. Weak approximation is known in fibers of good reduction [10], so we take simultaneous resolutions of singular fibers of $\mathcal{X}$ whenever possible. (For example, for rational double points on surfaces, simultaneous resolution is possible provided the local monodromy is trivial $[3,4]$.) Consequently, when $\mathcal{X} \rightarrow B$ admits a simultanteous resolution over some étale neighborhood of $b$, we replace $\mathcal{X}$ by this resolution. However, the resolved family may be an algebraic space, rather than a scheme, over $B$. This is why Theorem 1 is stated in this generality.

There are very few instances where weak approximation over function fields is known at all places: stably rational varieties; connected linear algebraic groups 
and their homogeneous spaces; homogeneous space fibrations over varieties that satisfy weak approximation, including conic bundles over rational varieties; and Del Pezzo surfaces of degree at least four [5]. Recently, the case of smooth hypersurfaces of degree $d$ in $\mathbb{P}^{n}$ with $d^{2} \leq n$ has been resolved [6], as an application of the notion of 'rational simply connectedness'. Even the case of cubic surfaces remains open, in general. Madore established weak approximation for cubic surfaces at places of good reduction [18]. His proof uses the abundance of distinct unirational parametrizations, and builds on ideas of Swinnerton-Dyer [21].

When is Theorem 1 applicable? Let $X$ be a smooth projective rationally connected variety over $F=k(B)$, with $B$ projective. There exists a regular proper model $\pi: \mathcal{X} \rightarrow B$, and any section $s: B \rightarrow \mathcal{X}$ is contained in $\mathcal{X}^{s m}$. For each singular fiber $\mathcal{X}_{b}$, fix an irreducible component $\mathcal{X}_{b}^{\bullet} \subset \mathcal{X}_{b}^{s m}$; these determine an open subset $\mathcal{X}^{\bullet} \subset \mathcal{X}^{s m}$. To prove weak approximation for $X$, it suffices to prove approximation for each $\mathcal{X}^{\bullet}$ obtained in this way. We do not know how to verify (1) in general: Is there any section meeting a prescribed irreducible component of $\mathcal{X}_{b}^{s m}$ ? Further, there is no general result giving a regular proper model $\mathcal{X} \rightarrow B$ such that each irreducible component of $\mathcal{X}_{b}^{s m}$ has the property (2).

Section 5 is devoted to applications to cubic surfaces:

Theorem 2. Let $X$ be a smooth cubic surface over $F$ and $\pi: \mathcal{X} \rightarrow B$ a proper model whose singular fibers are cubic surfaces with rational double points. Suppose there exists a section $s: B \rightarrow \mathcal{X}^{s m}$. Then sections of $\mathcal{X}^{s m} \rightarrow B$ satisfy approximation away from $S$.

When the model is regular all sections are contained in the smooth locus, so we conclude:

Corollary 3. Let $X$ be a smooth cubic surface over $F$. Suppose $X$ admits a regular proper model $\pi: \mathcal{X} \rightarrow B$ whose singular fibers are cubic surfaces with rational double points. Then weak approximation holds for $X$ away from $S=$ $\bar{B} \backslash B$.

There exist cubic surfaces which do not admit models with at most rational double points in a given fiber, e.g., the isotrivial family

$$
x^{3}+y^{3}+z^{3}=t w^{3}
$$


over the $t$-line. Nonetheless, Corollary 3 proves weak approximation for 'generic' cubic surfaces.

Corollary 4. Let $\mathcal{H i l b}=\mathbb{P}\left(\Gamma\left(\mathcal{O}_{\mathbb{P}^{3}}(3)\right)\right) \simeq \mathbb{P}^{19}$ denote the Hilbert scheme of cubic surfaces, $\mathcal{U} \rightarrow \mathcal{H}$ ilb the universal family, and $D \subset \mathcal{H}$ ilb the discriminant divisor. Let $B \subset \mathcal{H i l b}$ be a smooth curve transverse to each branch of $D$ and

$$
\mathcal{X}:=\mathcal{U} \times \mathcal{H i l b} B \rightarrow B
$$

the corresponding family. Then $X=\mathcal{U} \times \mathcal{H}_{\text {Hilb }} \operatorname{Spec}(F)$ satisfies weak approximation away from $S=\bar{B} \backslash B$.

Note that meeting the discriminant transversally is an open condition on the classifying map to the Hilbert scheme. This can be expressed in number-theoretic terms: The discriminant of $\mathcal{X} \rightarrow B$ is square-free. Generalizations to degree-two del Pezzo surfaces have been proven by Knecht [12].

Finally, in Section 6 we offer extensions of these Corollaries. We generalize Corollary 3 to the case where the fibers have isolated complete-intersection terminal singularities. Corollary 4 extends to generic hypersurfaces $X \subset \mathbb{P}^{n}$ of degree $d \leq n$ provided $n \geq 4$.

In our approach to approximation, we require precise control over proper rational curves in the smooth locus of a singular variety. One focus of this paper is to extend standard results on smooth proper rationally connected varieties to the non-proper case (see Section 4). The application to cubic surfaces and higherdimensional hypersurfaces involves refining rational connectivity results of [11] (see Sections 5 and 6).

Acknowledgments: We are grateful to J. L. Colliot-Thélène for numerous discussions about the problems considered here; the ideas here were developed during visits to Orsay by both authors. J. McKernan suggested the extensions to higher-dimensional hypersurfaces. We also benefitted from conversations with S. Keel, A. Knecht, and J. Kollár. The first author was partially supported by the Sloan Foundation and NSF Grants 0134259 and 0196187. The second author was partially supported by National Science Foundation Grants 0554280 and 0602333. 


\section{Notions of approximation}

Let $F$ be a global field, i.e., a number field or the function field of a curve $B$ defined over an algebraically closed field $k$. Let $S$ a finite set of places of $F$ containing the archimedean places, $\mathfrak{o}_{F, S}$ the corresponding ring of integers, and $\mathbb{A}_{F, S}$ the restricted direct product over all places outside $S$.

Let $X$ be an algebraic variety over $F, X(F)$ the set of $F$-rational points and $X\left(\mathbb{A}_{F, S}\right) \subset \prod_{v \notin S} X\left(F_{v}\right)$ the set of $\mathbb{A}_{F, S}$-points of $X$. The set $X\left(\mathbb{A}_{F, S}\right)$ carries a natural direct product topology. One says that weak approximation holds for $X$ away from $S$ if $X(F)$ is dense in this topology.

The set $X\left(\mathbb{A}_{F, S}\right)$ also carries a natural adelic topology: The basic open subsets are

$$
\prod_{v \in S^{\prime}} \mathfrak{u}_{v} \times \prod_{v \notin\left(S \cup S^{\prime}\right)} \mathcal{X}\left(\mathfrak{o}_{v}\right)
$$

where $S^{\prime}$ is a finite set of nonarchimedean places disjoint from $S, \mathcal{X} \rightarrow \operatorname{Spec}\left(\mathfrak{o}_{F, S}\right)$ is a model of $X$ (i.e., flat with generic fiber $X$ ), $\mathfrak{o}_{v}$ is the completion of $\mathfrak{o}_{F, S}$ at $v$, and $\mathfrak{u}_{v} \subset X\left(F_{v}\right)$ an open subset in the $v$-adic analytic topology on $X\left(F_{v}\right)$. This depends only on $X$ not on the choice of model. Strong approximation holds for $X$ away from $S$ if $X(F)$ is dense in $X\left(\mathbb{A}_{F, S}\right)$. Note that strong approximation implies weak approximation. Conversely, for $\mathcal{X} \rightarrow \operatorname{Spec}\left(\mathfrak{o}_{F, S}\right)$ flat and proper, weak approximation implies strong approximation, since $\mathcal{X}\left(\mathfrak{o}_{v}\right)=X\left(F_{v}\right)$; in these cases, we will use the term weak approximation for the sake of consistency.

Finally, there is a formulation which is sensitive to the choice of model. Consider the topology on $\prod_{v \notin S} \mathcal{X}\left(\mathfrak{o}_{v}\right)$ with basic open subsets

$$
\prod_{v \in S^{\prime}} \mathfrak{u}_{v} \times \prod_{v \notin\left(S \cup S^{\prime}\right)} \mathcal{X}\left(\mathfrak{o}_{v}\right)
$$

with $\mathfrak{u}_{v} \subset \mathcal{X}\left(\mathfrak{o}_{v}\right)$ an open subset. We say that approximation holds for $S$-integral points of $\mathcal{X}$ if $\mathcal{X}\left(\mathfrak{o}_{F, S}\right)$ is dense in this product. This is a version of strong approximation for integral points.

We now focus on the function field case: Let $\bar{B}$ be a smooth projective model of $B$ with $S=\bar{B} \backslash B$; places $v$ correspond to points $b \in \bar{B}$. Let $X$ be a smooth variety proper over $F=k(B), \pi: \mathcal{X} \rightarrow B$ a model proper and flat over $B$ (which exists by [20]), and $\mathcal{X}^{\bullet} \subset \mathcal{X}^{s m}$ an open subset surjecting onto $B$. Since $\pi$ is 
proper, $F$-rational points of $X$ correspond to sections $s: B \rightarrow \mathcal{X}$. If $\mathcal{X}$ is regular $s$ factors through $\mathcal{X}^{s m}$.

Definition 5. An admissible section of $\pi: \mathcal{X} \rightarrow B$ is a section $s: B \rightarrow \mathcal{X}^{s m}$. An admissible $N$-jet of $\pi$ at $b$ is a section of

$$
\mathcal{X}^{s m} \times_{B} \operatorname{Spec}\left(\mathcal{O}_{B, b} / \mathfrak{m}_{B, b}^{N+1}\right) \rightarrow \operatorname{Spec}\left(\mathcal{O}_{B, b} / \mathfrak{m}_{B, b}^{N+1}\right) .
$$

An approximable $N$-jet of $\pi$ at $b$ is a section of

$$
\mathcal{X} \times_{B} \operatorname{Spec}\left(\mathcal{O}_{B, b} / \mathfrak{m}_{B, b}^{N+1}\right) \rightarrow \operatorname{Spec}\left(\mathcal{O}_{B, b} / \mathfrak{m}_{B, b}^{N+1}\right)
$$

that may be lifted to a section of $\widehat{\mathcal{X}}_{b} \rightarrow \widehat{B}_{b}$, with $\widehat{B}_{b}=\operatorname{Spec}\left(\hat{\mathcal{O}}_{B, b}\right)$ and $\widehat{\mathcal{X}}_{b}=$ $X \times_{B} \widehat{B}_{b}$.

Hensel's lemma guarantees that every admissible $N$-jet is approximable. Let $\left\{b_{i}\right\}_{i \in I}$ be a finite set of points and $j_{i}$ an admissible $N$-jet of $\pi$ at $b_{i}$. We write $J=\left\{j_{i}\right\}_{i \in I}$ for the corresponding collection of admissible $N$-jets.

The notions of weak and strong approximation introduced above have geometric interpretations

- Weak and strong approximation hold for $X$ away from $S$ if any finite collection of approximable jets of $\pi$ can be realized by a section $s: B \rightarrow \mathcal{X}$.

- This is equivalent to weak approximation holding for $X^{\bullet}$ (the generic fiber of $\mathcal{X}^{\bullet}$ ) away from $S$ : Every jet in $\mathcal{X}$ at $b$ can be realized by a section $\mathcal{X} \times_{B} \widehat{B}_{b} \rightarrow \widehat{B}_{b}$ meeting $\widehat{\mathcal{X}}_{b}^{\bullet}$.

- If $\mathcal{X}$ is regular these are equivalent to the condition that any collection of admissible jets of $\pi$ can be realized by a section $s: B \rightarrow \mathcal{X}^{s m}$.

There is an analogous formulation of approximation for integral points:

- Approximation holds for sections of $\mathcal{X}^{\bullet} \rightarrow B$ away from $S$ if each collection of jet data in $\mathcal{X}^{\bullet}$ can be realized by a section $s: B \rightarrow \mathcal{X}^{\bullet}$.

- If $\mathcal{X}$ is regular and $\mathcal{X}^{\bullet}=\mathcal{X}^{s m}$ this is equivalent to weak approximation for $X$. 


\section{Curves, combs, and deformations}

The dual graph associated with a nodal curve $C$ has vertices are indexed by the irreducible components of $C$ and its edges indexed by the intersections of these components. A projective nodal curve $C$ is tree-like if

- each irreducible component of $C$ is smooth;

- the dual graph of $C$ is a tree.

Definition 6. A comb with $m$ reducible teeth is a projective nodal curve $C$ with $m+1$ subcurves $D, T_{1}, \ldots, T_{m}$ such that

- $D$ is smooth and irreducible;

- $T_{l} \cap T_{l^{\prime}}=\emptyset$, for all $l \neq l^{\prime}$;

- each $T_{l}$ meets $D$ transversally in a single point; and

- each $T_{l}$ is a chain of $\mathbb{P}^{1}$ 's.

Here $D$ is called the handle and the $T_{l}$ the reducible teeth.

Let $C$ be a nodal curve and $h: C \rightarrow W$ an immersion into a smooth algebraic space with nodal image. (In particular, $h$ is an embedding at nodes of $C$.) Let $\mathcal{N}_{h}$ denote the normal bundle (or sheaf), i.e., the dual to the kernel of the restriction $h^{*} \Omega_{W}^{1} \rightarrow \Omega_{C}^{1}$.

We will use the following lemma, which has the same proof as Proposition 24 of [10]:

Lemma 7. Let $C$ be a tree-like curve, $W$ a smooth algebraic space, $h: C \rightarrow W$ an immersion with nodal image. Suppose that for each irreducible component $C_{l}$ of $C, H^{1}\left(C_{l}, \mathcal{N}_{h} \otimes \mathcal{O}_{C_{l}}\right)=0$ and $\mathcal{N}_{h} \otimes \mathcal{O}_{C_{l}}$ is globally generated. Then $h$ deforms to an immersion of a smooth curve into $W$.

Suppose furthermore that $\mathfrak{w}=\left\{w_{1}, \ldots, w_{M}\right\} \subset C$ is a collection of smooth points such that for each component $C_{l}, H^{1}\left(\mathcal{N}_{h} \otimes \mathcal{O}_{C_{l}}(-\mathfrak{w})\right)=0$ and the sheaf $\mathcal{N}_{h} \otimes \mathcal{O}_{C_{l}}(-\mathfrak{w})$ admits a section nonvanishing at each point of the support of

$$
\left(\mathcal{N}_{h} \otimes \mathcal{O}_{C_{l}}\right) / \mathcal{N}_{h \mid C_{l}}
$$

Then $h: C \rightarrow W$ deforms to an immersion of a smooth curve into $W$ containing $h(\mathfrak{w})$. 


\section{Strong Rational Connectivity}

Definition 8. A variety $X$ is rationally connected (resp. separably rationally connected) if there is a family of proper irreducible rational curves $g: U \rightarrow Z$ (resp. $\pi_{2}: U=\mathbb{P}^{1} \times Z \rightarrow Z$ ) and a cycle morphism $u: U \rightarrow X$ such that

$$
u^{2}: U \times{ }_{Z} U \rightarrow X \times X
$$

is dominant (resp. smooth over the generic point)).

Intuitively, two generic points of $X$ can be joined by an irreducible projective rational curve. Over fields of characteristic zero, rational connected varieties are also separably rationally connected [15] IV.3.3.1.

The notion of rational connectedness is a bit subtle over countable fields [2]. For convenience, we work over an uncountable algebraically closed field. Over such a field, rational connectivity is equivalent to the condition that two very general points of $X$ can be joined by such a rational curve.

Definition 9. Let $X$ be a smooth algebraic space of dimension $d$ and $f: \mathbb{P}^{1} \rightarrow X$ a nonconstant morphism, so we have an isomorphism

$$
f^{*} \mathcal{T}_{X} \simeq \mathcal{O}_{\mathbb{P}^{1}}\left(a_{1}\right) \oplus \ldots \oplus \mathcal{O}_{\mathbb{P}^{1}}\left(a_{d}\right)
$$

for suitable integers $a_{1}, \ldots, a_{d}$. Then $f$ is free (resp. very free) if each $a_{i} \geq 0$ (resp. $a_{i} \geq 1$ ).

We refer the reader to [15] IV.3 for further facts about rationally connected varieties.

One technical result will play a prominent rôle in our analysis.

Proposition 10 ([15] IV.3.9.4). Let $V$ be a smooth separably rationally connected (not necessarily proper) variety. Then there exists a nonempty subset $V^{0} \subset V$ characterized as the largest open subset such that if $v_{1}, \ldots, v_{m} \in V^{0}$ are distinct closed points, then there is a very free curve in $V^{0}$ containing these as smooth points. Moreover, any rational curve $C \subset V$ that meets $V^{0}$ is contained in $V^{0}$.

No example where $V^{0} \neq V$ is known.

Remark 11. Let $V_{2}$ be a smooth variety, $V_{1} \subset V_{2}$ a rationally connected dense open subvariety, and $V_{2}^{0} \subset V_{2}$ the largest open set satisfying the conditions of 
Proposition 10 . Then $V_{1}^{0} \subset V_{2}^{0}$. Thus a point $v \in V_{2}$ is in $V_{2}^{0}$ provided there is a rational curve $f: \mathbb{P}^{1} \rightarrow V_{2}$ through $v$ and meeting $V_{1}^{0}$.

Proposition 12. Let $V$ be a smooth separably rationally connected variety, and $\beta: W \rightarrow V$ an iterated blow-up of $V$ along smooth subvarieties. Then $\beta^{-1}\left(V^{0}\right)=$ $W^{0}$.

Proof. The inclusion $W^{0} \subset \beta^{-1}\left(V^{0}\right)$ is straightforward: Given points $w_{1}, \ldots, w_{m} \in$ $W^{0}$, there is a very free curve $g: \mathbb{P}^{1} \rightarrow W^{0}$ containing them; we may choose this to be transversal to the exceptional divisor of $\beta$. The inclusion of sheaves

$$
\mathcal{T}_{W} \hookrightarrow \beta^{*} \mathcal{T}_{V}
$$

remains an inclusion after pull-back via $g$, as the support of the cokernel does not contain $g\left(\mathbb{P}^{1}\right)$. The positivity of $g^{*} \mathcal{T}_{W}$ implies the positivity of $(\beta \circ g)^{*} \mathcal{T}_{V}$, which means that $\beta \circ g: \mathbb{P}^{1} \rightarrow V$ is also very free.

For the reverse direction, we may restrict to the case where $W$ is the blow-up of $V$ along a smooth subvariety $Z$ of codimension $r>1$, with exceptional divisor $E$. It is clear that $\beta^{-1}\left(V^{0} \backslash Z\right) \subset W_{0}$, so consider some $w \in \beta^{-1}(z)$ with $z \in Z \cap V^{0}$. It suffices to construct a rational curve containing $w$ and the generic point of $W$.

There exists a very free curve $f^{\prime}: \mathbb{P}^{1} \rightarrow V^{0}$ with the following properties:

(1) $f^{\prime}\left(\mathbb{P}^{1}\right)$ meets $Z$ only at $z$ (we can always deform a very free curve to a curve passing through $z$ and disjoint from a codimension $\geq 2$ subset $[15$, II.3.7]);

(2) $f^{\prime}\left(\mathbb{P}^{1}\right)$ is smooth at $z$ and transverse to $Z$.

Let $g^{\prime}: \mathbb{P}^{1} \rightarrow W$ denote the lift to $W$, which is free in $W$, and $w^{\prime}=g^{\prime}(0)$. If $w^{\prime}=w$ then we are done. Otherwise, let $\ell \subset \beta^{-1}(z) \simeq \mathbb{P}^{r-1}$ denote the line joining $w$ and $w^{\prime}$. Since $g^{\prime}$ is free, it admits a small deformation to a free curve $g^{\prime \prime}: \mathbb{P}^{1} \rightarrow W$ with $w^{\prime \prime}:=g^{\prime \prime}(0) \in \ell, w^{\prime \prime} \neq w^{\prime}$. (See Figure 1.)

We construct a comb $h: C \rightarrow W$ with handle $\ell \subset \mathbb{P}^{r-1} \subset W$ and two teeth $g^{\prime}, g^{\prime \prime}: \mathbb{P}^{1} \rightarrow W$. Since $E$ is exceptional, we compute

$$
\mathcal{N}_{\ell / E} \simeq \mathcal{O}_{\mathbb{P}^{1}}^{\operatorname{dim}(V)} \oplus \mathcal{O}_{\mathbb{P}^{1}}(1)^{r-2}
$$

and

$$
\mathcal{N}_{E / W} \otimes \mathcal{O}_{\ell} \simeq \mathcal{O}_{\mathbb{P}^{1}}(-1)
$$




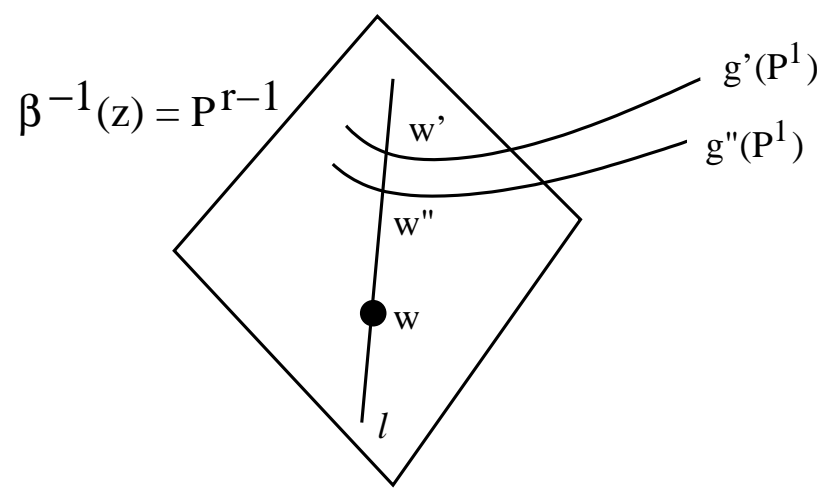

FiguRE 1. Constructing the comb

The exact sequence of normal bundles

$$
0 \rightarrow \mathcal{N}_{\ell / E} \rightarrow \mathcal{N}_{\ell / W} \rightarrow \mathcal{N}_{E / W} \otimes \mathcal{O}_{\ell} \rightarrow 0
$$

splits because

$$
\operatorname{Ext}^{1}\left(\mathcal{O}_{\mathbb{P}^{1}}(-1), \mathcal{O}_{\mathbb{P}^{1}}(n)\right)=0, n \geq-2 .
$$

Thus we deduce

$$
\mathcal{N}_{\ell / W} \simeq \mathcal{O}_{\mathbb{P}^{1}}^{\operatorname{dim}(V)-r} \oplus \mathcal{O}_{\mathbb{P}^{1}}(1)^{r-2} \oplus \mathcal{O}_{\mathbb{P}^{1}}(-1)
$$

where the negative summand is in the normal direction to $E$. Since $g^{\prime}\left(\mathbb{P}^{1}\right)$ and $g^{\prime \prime}\left(\mathbb{P}^{1}\right)$ are transverse to $E$, we can apply Proposition 23 of [10]. The key point is that the only negativity in the normal bundle of $\ell$ is due to the negativity of the normal bundle of $E \subset W$; however, the components $g^{\prime}\left(\mathbb{P}^{1}\right)$ and $g^{\prime \prime}\left(\mathbb{P}^{1}\right)$ overcome this. Precisely, we have

$$
\mathcal{N}_{h} \otimes \mathcal{O}_{\ell} \simeq \mathcal{O}_{\mathbb{P}^{1}}^{\operatorname{dim}(V)-r} \oplus \mathcal{O}_{\mathbb{P}^{1}}(1)^{r-2} \oplus \mathcal{O}_{\mathbb{P}^{1}}(1) ;
$$

the quotient $\left(\mathcal{N}_{h} \otimes \mathcal{O}_{\ell}\right) / \mathcal{N}_{\ell / W}$ lies in the image of the last summand.

Lemma 7 implies that $h: C \rightarrow W$ admits a deformation to a rational curve containing $w$.

A similar argument gives the following strengthening of Proposition 10 (cf. Theorem 2.2 of [7])

Proposition 13. Let $V$ be a smooth separably rationally connected variety and $V^{0} \subset V$ be the distinguished open subset characterized in Proposition 10. Then 
for any finite collection of jets

$$
j_{i}: \operatorname{Spec}\left(k[\epsilon] /\left\langle\epsilon^{N+1}\right\rangle\right) \hookrightarrow V^{0}, \quad i=1, \ldots, m
$$

supported at distinct points $v_{1}, \ldots, v_{m}$, there exists a very free rational curve smooth at $v_{1}, \ldots, v_{m}$ with the prescribed jets.

Proof. There is an iterated blow-up

$$
\beta: W=W_{N} \rightarrow \ldots \rightarrow W_{j} \rightarrow \ldots \rightarrow W_{1} \rightarrow V
$$

and points $w_{1}, \ldots, w_{m} \in W$ so that if $g: C \rightarrow W$ is a morphism whose image contains $w_{1}, \ldots, w_{m}$ then the image of $f:=\beta \circ g: C \rightarrow V$ contains the given collection of jets. Here is the description: Over each point $v_{i}$, we blow up $V$ successively at $N$ points. Given any smooth curve germ $C$ with the prescribed $N$-jet at $v_{i}, W_{j}$ is the blowup of $W_{j-1}$ at the points of the proper transform of $C$ lying over the $v_{i}$. Proposition 12 then implies there exists a very free curve $g: \mathbb{P}^{1} \rightarrow W$ through $w_{1}, \ldots w_{m}$. However, the image of this curve in $V$ will be singular at $v_{i}$ if $g\left(\mathbb{P}^{1}\right)$ meets $\beta^{-1}\left(v_{i}\right)$ in more than one point.

We claim there exists a very free curve $g_{i}: \mathbb{P}^{1} \rightarrow W$ meeting $\beta^{-1}\left(v_{i}\right)$ only at $w_{i}$, transversally. We choose this curve so that it is disjoint from $\beta^{-1}\left(v_{j}\right)$ when $j \neq i$. (Again, we are using the fact that a very free curve can be deformed away from any codimension $\geq 2$ subvariety while passing through a prescribed point in the complement.) Fix generic points $x_{i} \in g_{i}\left(\mathbb{P}^{1}\right)$ and let $g_{0}: \mathbb{P}^{1} \rightarrow W$ be a very free curve intersecting $g_{i}\left(\mathbb{P}^{1}\right)$ transversely at $x_{i}$ but not meeting any $\beta^{-1}\left(v_{i}\right)$. (For example, take $g_{0}=\left(\beta^{-1} \circ f_{0}\right)$, where $f_{0}: \mathbb{P}^{1} \rightarrow V$ is a very free curve through $\beta\left(x_{1}\right), \ldots, \beta\left(x_{m}\right)$.) Consider the comb $h: C \rightarrow W$ with handle $g_{0}\left(\mathbb{P}^{1}\right)$ and $m$ teeth $g_{i}\left(\mathbb{P}^{1}\right)$. This deforms to a very free curve $h^{\prime}: \mathbb{P}^{1} \rightarrow W$ meeting each $\beta^{-1}\left(v_{i}\right)$ only at $w_{i}$, transversally.

The proof of the claim is a refinement of the argument for Proposition 12 . We proceed by induction on $N$. The base case $N=1$ is contained in the proof of Proposition 12, which gives a very free curve smooth at $v_{i}$ with prescribed tangency. Let $E_{i, N} \simeq \mathbb{P}^{\operatorname{dim}(V)-1}$ be the last exceptional divisor of $\beta: W \rightarrow V$ over $v_{i}$, i.e., the exceptional divisor of the $N$-th blow-up. For $1 \leq j<N$, let $E_{i, j} \subset W_{N}$ denote the proper transform of the exceptional divisor of $W_{j} \rightarrow W_{j-1}$ over $v_{i}$; we have $E_{i, j} \simeq \mathrm{Bl}_{w_{i, j}} \mathbb{P}^{\operatorname{dim}(V)-1}$, where $w_{i, j}$ is the intersection of the proper transform of $C$ with the exceptional divisor of $W_{j} \rightarrow W_{j-1}$. 
Suppose that $g_{i}^{\prime}: \mathbb{P}^{1} \rightarrow W$ is a very free curve such that $\beta \circ g_{i}^{\prime}$ is smooth with the desired $(N-1)$-jet at $v_{i}$. Let $w_{i}^{\prime}=g_{i}^{\prime}\left(\mathbb{P}^{1}\right) \cap \beta^{-1}\left(v_{i}\right)$ denote the unique point of intersection, which we assume is distinct from $w_{i}$. Let $\ell_{N}$ denote the line in $E_{i, N} \simeq \mathbb{P}^{\operatorname{dim}(V)-1}$ joining $w_{i}$ and $w_{i}^{\prime}$, and $z_{N-1}$ its point of intersection with $E_{i, N-1}$. Let $\ell_{N-1} \subset E_{i, N-1} \simeq \mathrm{Bl}_{w_{i, N-1}} \mathbb{P}^{\operatorname{dim}(V)-1}$ denote the proper transform of a line containing $z_{N-1}$, and $z_{N-2}$ its point of intersection with $E_{i, N-2}$. Continue in this way, until we obtain $\ell_{1} \subset E_{i, 1}$, the proper transform of a line containing $z_{1}$. Finally, let $g_{i}^{\prime \prime}: \mathbb{P}^{1} \rightarrow W$ be a very free curve meeting the exceptional locus transversally at a generic point of $\ell_{1}$. (See Figure 2.)

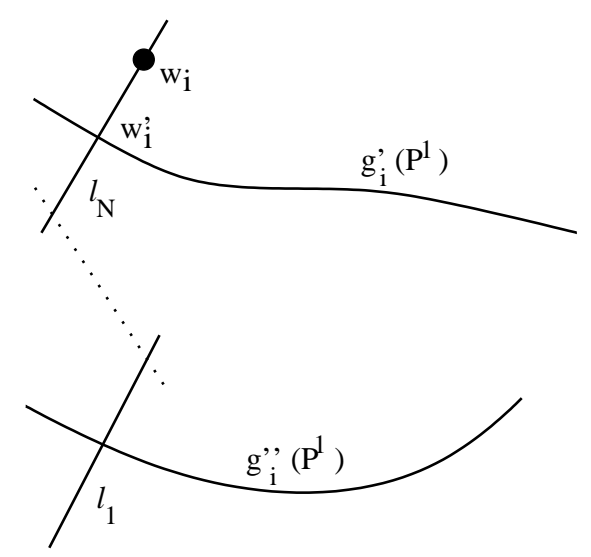

FiguRE 2. Constructing the comb with reducible teeth

Let $h: C \rightarrow W$ be the comb with handle $\ell_{N}$ and two reducible teeth:

(1) $g_{i}^{\prime}: \mathbb{P}^{1} \rightarrow W$

(2) the union of the lines $\ell_{N-1}, \ldots, \ell_{1}$ and the curve $g_{i}^{\prime \prime}: \mathbb{P}^{1} \rightarrow W$;

By a normal bundle computation similar to that of Proposition 12, we find that $\mathcal{N}_{h} \mid \ell_{N}$ is ample and $\mathcal{N}_{h}$ is nonnegative on each of the remaining components: Again, Lemma 7 (or Proposition 24 of [10]) implies that $h$ admits a deformation to an immersed rational curve containg $w_{i}$.

Here are the details of the computations (cf. [10] Section 5): The normal bundle of a line in projective space is

$$
\mathcal{N}_{\ell_{N} / E_{i, N}}=\mathcal{N}_{\ell_{N} / \mathbb{P} \operatorname{dim}(V)-1} \simeq \mathcal{O}_{\mathbb{P}^{1}}(1)^{\operatorname{dim}(V)-2}
$$


and the normal bundle for an exceptional divisor is

$$
\mathcal{N}_{E_{i, N} / W} \simeq \mathcal{O}_{\mathbb{P} \operatorname{dim}(V)-1}(-1) .
$$

For each $j$ we have

$$
\left.0 \rightarrow \mathcal{N}_{\ell_{j} / E_{i, j}} \rightarrow \mathcal{N}_{\ell_{j} / W} \rightarrow \mathcal{N}_{E_{i, j} / W}\right|_{\ell_{j}} \rightarrow 0
$$

which for $j=N$ yields

$$
\mathcal{N}_{\ell_{N} / W} \simeq \mathcal{O}_{\mathbb{P}^{1}}(1)^{\operatorname{dim}(V)-2} \oplus \mathcal{O}_{\mathbb{P}^{1}}(-1),
$$

with the negative component in the direction normal to $E_{i, N}$. We also have an extension

$$
\left.0 \rightarrow \mathcal{N}_{\ell_{j} / W} \rightarrow \mathcal{N}_{h}\right|_{\ell_{j}} \rightarrow Q\left(\ell_{j}\right) \rightarrow 0
$$

where $Q\left(\ell_{j}\right)$ is a torsion sheaf supported at the points where $\ell_{j}$ meets the adjacent components. For $j=N$ these are $g_{i}^{\prime}\left(\mathbb{P}^{1}\right)$ and $\ell_{N-1}$, and since the tangent vectors to these curves are normal to $E_{i, N}$, we find

$$
\left.\mathcal{N}_{h}\right|_{\ell_{N}} \simeq \mathcal{O}_{\mathbb{P}^{1}}(1)^{\operatorname{dim}(V)-2} \oplus \mathcal{O}_{\mathbb{P}^{1}}(1)
$$

The normal bundle of the proper transform of a line in the blow-up of projective space at a point of the line is

$$
\mathcal{N}_{\ell_{j} / E_{i, j}}=\mathcal{N}_{\ell_{j} / \mathrm{Bl}_{w_{i, j}} \mathbb{P}^{\operatorname{dim}(V)-1}} \simeq \mathcal{O}_{\mathbb{P}^{1}}^{\operatorname{dim}(V)-2}
$$

for $j=1, \ldots, N-1$. Similarly, we can compute

$$
\left.\mathcal{N}_{E_{i, h} / W}\right|_{\ell_{j}}=\mathcal{O}_{\mathbb{P}^{1}}(-2)
$$

so the exact sequence analogous to (4.1) yields

$$
\mathcal{N}_{\ell_{j} / W} \simeq \mathcal{O}_{\mathbb{P}^{1}}^{\operatorname{dim}(V)-2} \oplus \mathcal{O}_{\mathbb{P}^{1}}(-2),
$$

with the negative component in the direction normal to $E_{i, j}$. Using (4.2) and the fact that $\ell_{j}$ is adjacent to $\ell_{j+1}$ and $\ell_{j-1}\left(\right.$ or $g_{i}^{\prime \prime}\left(\mathbb{P}^{1}\right)$ when $\left.j=1\right)$, we find

$$
\left.\mathcal{N}_{h}\right|_{\ell_{j}} \simeq \mathcal{O}_{\mathbb{P}^{1}}^{\operatorname{dim}(V)-2} \oplus \mathcal{O}_{\mathbb{P}^{1}}
$$

Definition 14. A smooth separably rationally connected variety $Y$ is strongly rationally connected if any of the following conditions hold:

(1) for each point $y \in Y$, there exists a rational curve $f: \mathbb{P}^{1} \rightarrow Y$ joining $y$ and a generic point in $Y$; 
(2) for each point $y \in Y$, there exists a free rational curve containing $y$;

(3) for any finite collection of points $y_{1}, \ldots, y_{m} \in Y$, there exists a very free rational curve containing the $y_{j}$ as smooth points;

(4) for any finite collection of jets

$$
\operatorname{Spec}\left(k[\epsilon] /\left\langle\epsilon^{N+1}\right\rangle\right) \subset Y, i=1, \ldots, m
$$

supported at distinct points $y_{1}, \ldots, y_{m}$, there exists a very free rational curve smooth at $y_{1}, \ldots, y_{m}$ and containing the prescribed jets.

The implications

$$
(4) \Rightarrow(3) \Rightarrow(2) \Rightarrow(1)
$$

are obvious. By Proposition 10, assertions (1)-(3) are each equivalent to the condition $Y=Y^{0}$. Property (4) is analogous to Theorem 2.2 of [7], which is stated for proper varieties. It follows from (1) by Proposition 13.

With basic properties of strongly rationally connected varieties established, Theorem 1 follows from the general result (cf. [15] IV.6.10.1):

Theorem 15. Let $\pi: \mathcal{Y} \rightarrow B$ be a smooth morphism whose fibers are strongly rationally connected. Assume that $\pi$ has a section. Then sections of $\mathcal{Y} \rightarrow B$ satisfy approximation away from $S$.

Proof. Let $\bar{\pi}: \overline{\mathcal{Y}} \rightarrow \bar{B}$ be a proper model of $\mathcal{Y} \rightarrow B$, which exists by [20]. The section extends to a section $\bar{s}$ of $\bar{\pi}$. By a result of Artin and Néron [1] Corollary 4.6, there exists a blow-up with center supported in $\bar{\pi}^{-1}(S)$

$$
\widetilde{\mathcal{Y}} \rightarrow \overline{\mathcal{Y}}
$$

such that the proper transform of $\bar{s}(\bar{B})$ in $\tilde{\mathcal{Y}}$ is contained in $\widetilde{\mathcal{Y}}^{s m}$.

Recall the proof of weak approximation at places of good reduction in Section 5 of [10]. This is a bootstrap argument, using the existence of a section in the smooth locus to construct sections with prescribed jets of successively higher order. For the base case, suppose we are given an arbitrary section $t: B \rightarrow \mathcal{Y}$. When $\mathcal{Y} \rightarrow B$ is proper, Kollár-Miyaoka-Mori [16] first demonstrated how to get a section with prescribed values $y_{1}, \ldots, y_{r}$ at $b_{1}, \ldots, b_{r} \in B$. The key is to construct a comb with handle $t(B)$ and teeth very free curves in the fibers $\mathcal{Y}_{b_{i}}$ joining $t\left(b_{i}\right)$ to $y_{i}$, which deforms to a section passing through the $y_{i}$. For the inductive step, 
suppose we have a section with prescribed jets to order $\leq N-1$ at a finite set of points $b_{1}, \ldots, b_{r}$. Blow up the total space $N$ times along the jet data at each of $b_{1}, \ldots, b_{r}$ to get a new model with (admittedly very special) reducible fibers over $b_{1}, \ldots, b_{r}$. The $N$ th-order jet conditions in the original model translate into point conditions in the new model. There we produce an explicit comb with reducible teeth, based on the proper transform of the section obtained by the inductive hypothesis, that deforms to the desired section. This only requires the existence of very free curves in $\mathcal{Y}_{b_{i}}$ passing through $y_{i}$ with prescribed tangency.

Properness is used twice. At the the zeroth-order step, it is used to exhibit the very free fibral curves joining $t\left(b_{i}\right)$ to $y_{i}$. In the inductive step, it is used to find a very free fibral curve with prescribed tangency at $y_{i}$. In our situation, these are guaranteed by the hypothesis that the fibers are strongly rationally connected.

\section{Cubic surfaces}

We work over an algebraically closed field of characteristic zero.

Definition 16. A log Del Pezzo surface is a pair $(X, \Delta)$ consisting of a normal projective surface $X$ and an effective $\mathbb{Q}$-divisor $\Delta=\sum a_{i} \Delta_{i}, 0<a_{i} \leq 1$ on $X$, with $\log$ terminal singularities, such that $-\left(K_{X}+\Delta\right)$ is ample. When $\Delta$ is empty, this is equivalent to saying that $X$ has quotient singularities and ample anticanonical class.

Theorem 17 ([11] 1.6). The smooth locus of a log Del Pezzo surface $(X, \Delta)$ is rationally connected, i.e., two generic points in $X^{\text {sm }}$ can be joined by an irreducible projective rational curve contained in $X^{s m}$.

Example 18 ([23]). There exist projective rational surfaces with rational double points whose smooth locus is not rationally connected. Consider

$$
\widetilde{X}=E \times \mathbb{P}^{1}
$$

where $(E, 0)$ is an elliptic curve and the involution

$$
\begin{aligned}
\iota: \tilde{X} & \rightarrow \widetilde{X} \\
\left(e,\left[x_{0}, x_{1}\right]\right) & \mapsto\left(-e,\left[x_{1}, x_{0}\right]\right) .
\end{aligned}
$$

The involution has eight isolated fixed points $\mathfrak{q} \subset \widetilde{X}$. The quotient $X=\widetilde{X} /\langle\iota\rangle$ has eight $A_{1}$ singularities and is rational: $X \rightarrow E /\langle\iota\rangle \simeq \mathbb{P}^{1}$ is a conic bundle. 
Since $\widetilde{X}-\mathfrak{q} \rightarrow X^{s m}$ is a covering space, $\pi_{1}\left(X^{s m}\right) \subset \pi_{1}(\widetilde{X}-\mathfrak{q})$ with index two. Thus

$$
\pi(\widetilde{X}-\mathfrak{q}) \simeq \pi(\widetilde{X}) \simeq \pi(E) \simeq \mathbb{Z} \times \mathbb{Z}
$$

and $X^{s m}$ has infinite fundamental group. However, rationally connected varieties (even non-proper ones) have finite fundamental groups (see Lemma 7.8 of [11] and Proposition 2.10 of [14], for example).

The following conjecture would allow us to apply Theorem 1 to prove weak approximation for many log Del Pezzo surfaces:

Conjecture 19. The smooth locus of a log Del Pezzo surface is strongly rationally connected.

We prove this for cubic surfaces:

Theorem 20. Let $X \subset \mathbb{P}^{3}$ be a cubic surface with rational double points. Then $X^{s m}$ is strongly rationally connected.

Proof. Let $x_{1} \in X^{s m}$ be a point. We produce a rational curve $R \subset X^{s m}$ joining $x_{1}$ and a generic point $x_{2} \in X^{s m}$.

We start with an elementary lemma:

Lemma 21. Let $Y \subset \mathbb{P}^{n}$ be an irreducible hypersurface such that the Gauss map

$$
\begin{aligned}
& Y \leftrightarrow \check{\mathbb{P}}^{n} \\
& y \mapsto\left[\left.\mathcal{T}_{Y}\right|_{y}\right]
\end{aligned}
$$

is generically finite. Then a generic tangent hyperplane section to $Y$ has an isolated singularity of multiplicity two with smooth projectivized tangent cone.

Proof. Since the Gauss map is generically finite, its differential is generically of maximal rank. However, the differential at $y \in Y$ can be identified with the dual to the second fundamental form (see $[9,17.11]$ )

$$
\Pi_{y}:\left.\operatorname{Sym}^{2}\left(\left.\mathcal{T}_{Y}\right|_{y}\right) \rightarrow \mathcal{N}_{Y / \mathbb{P}^{n}}\right|_{y} .
$$

This is nondegenerate precisely when the quadratic term of the Taylor expansion of the defining equation of the tangent hyperplane section $H_{y}$ has maximal rank. 
This is applicable to cubic surfaces $X$ with rational double points. It is a classical fact that $X$ contains a finite number of lines. However, if the image of the Gauss map of $X$ is a curve $C$ then $X$ is dual to $C$ and thus ruled by lines.

Now we will make explicit how $x_{2}$ must be chosen. Applying the lemma, we may assume

(1) The tangent hyperplane section $H_{2}$ at $x_{2}$ is irreducible and nodal.

In particular, $H_{2} \subset X^{s m}$ and there are no lines $\ell \subset X$ containing $x_{2}$.

Projection from $x_{2}$ then gives a double cover

$$
\mathrm{Bl}_{x_{2}} X \rightarrow \mathbb{P}^{2}
$$

the covering transformation interchanges the exceptional divisor and the proper transform. We obtain a birational involution

$$
\begin{aligned}
\iota_{x_{2}}: X & \rightarrow X \\
x & \mapsto x^{\prime},
\end{aligned}
$$

where $\left\{x, x^{\prime}, x_{2}\right\}$ are collinear. This factors as the blow-up of $x_{2}$ followed by the blow-down of the proper transform of $H_{2}$. Note that $\iota_{x_{2}}$ fixes the singularities of $X$ and thus takes $X^{s m}$ to itself.

We also assume:

(2) $H_{2}$ does not contain $x_{1}$.

It follows that $H_{2}$ does not contain $x_{1}^{\prime}=\iota_{x_{2}}\left(x_{1}\right)$. Moreover, $x_{1}$ and $x_{1}^{\prime}$ are in the open subset on which $\iota_{x_{2}}$ is an isomorphism.

We assume furthermore:

(3) $x_{2}$ is not contained in $H_{1}$, the tangent hyperplane to $X$ at $x_{1}$.

It follows that $x_{2} \notin H_{1}^{\prime}$, the tangent hyperplane section at $x_{1}^{\prime}$. Indeed, suppose that $x_{2} \in H_{1}^{\prime}$. We know that $x_{2} \neq x_{1}^{\prime}$ (because $x_{1}^{\prime} \notin H_{2}$ ), so consider the line joining $x_{2}$ and $x_{1}^{\prime}$. This meets $X$ only at $x_{2}$ and $x_{1}^{\prime}$, so $x_{1}^{\prime}=x_{1}$ and $x_{2} \in H_{1}$, a contradiction.

Finally, we assume:

(4) $H_{1}^{\prime}$ is irreducible and nodal. 
In particular, $H_{1}^{\prime} \subset X^{s m}$.

Since $x_{2} \notin H_{1}^{\prime}, \iota_{x_{2}}$ is regular along $H_{1}^{\prime}$. We verify that the rational curve $R=\iota_{x_{2}}\left(H_{1}^{\prime}\right)$ has the desired properties. Since $x_{2} \notin H_{1}^{\prime}, H_{2}$ and $H_{1}^{\prime}$ intersect at a point $z \neq x_{2}$; thus the curve

$$
R=\iota_{x_{2}}\left(H_{1}^{\prime}\right) \ni \iota_{x_{2}}(z)=x_{2} .
$$

We know $H_{1}^{\prime} \subset X^{s m}$ and $\iota_{x_{2}}\left(X^{s m}\right) \subset X^{s m}$, hence $R \subset X^{s m}$. We have $x_{1}^{\prime} \in H_{1}^{\prime}$, so $x_{1}=\iota_{x_{2}}\left(x_{1}^{\prime}\right) \in R$. Since $H_{1}^{\prime}$ meets $H_{2}$ in a point $z \neq x_{2}, x_{2}=\iota_{x_{2}}(y) \in R$.

We now prove Theorem 2: For each singular fiber $\mathcal{X}_{b}, \mathcal{X}_{b}^{s m}$ is strongly rationally connected by Theorem 20. Approximation follows from Theorem 1.

Example 22. Here is another case where Conjecture 19 is easily verified. Let $X$ be a partial resolution of a cubic surface $\Sigma$ with at most $A_{1}$-singularities, i.e., we have a factorization of the minimal resolution

$$
\widetilde{\Sigma} \rightarrow X \stackrel{\beta}{\rightarrow} \Sigma \text {. }
$$

Then $X^{s m}$ is strongly rationally connected.

Theorem 2 implies that $\Sigma^{s m}$ is strongly rationally connected, hence $\beta^{-1}\left(\Sigma^{s m}\right) \subset$ $\left(X^{s m}\right)^{0}$. The locus $X^{s m} \backslash \beta^{-1}\left(\Sigma^{s m}\right)$ is a union of $(-2)$-curves $\left\{E_{i}\right\}$, corresponding to the resolved singularities $\left\{p_{i}\right\}$ of $\Sigma$. If $\left(X^{s m}\right)^{0}$ meets $E_{i}$, it must also contain $E_{i}$. Hence it suffices to show that for each $E_{i}$ there exists a rational curve in $X^{s m}$ meeting $E_{i}$ and $\beta^{-1}\left(\Sigma^{s m}\right)$ (see Remark 11).

To find this rational curve, consider the projection from $p_{i}$

$$
\pi_{i}: \Sigma \rightarrow \mathbb{P}^{2}
$$

which induces a morphism $\pi_{i}^{\prime}: X \rightarrow \mathbb{P}^{2}$. The image of $E_{i}$ is a plane conic and the image of the singularities of $X$ has codimension two in $\mathbb{P}^{2}$, so there exists a rational curve

$$
f: \mathbb{P}^{1} \rightarrow \mathbb{P}^{2} \backslash \pi_{i}^{\prime}(\operatorname{Sing}(X))
$$

meeting the image of $E_{i}$.

The same argument applies if $X$ is obtained from a cubic surface $\Sigma$ with $A_{1}$ and $A_{2}$ singularities by resolving some subset of $\operatorname{Sing}(\Sigma)$.

Corollary 4 is an immediate consequence of Corollary 3 and the following: 
Lemma 23. Let $\mathcal{H i l b}=\mathbb{P}\left(\Gamma\left(\mathcal{O}_{\mathbb{P}^{n}}(d)\right)\right)$ denote the Hilbert scheme of degree-d hypersurfaces, $\mathcal{U} \rightarrow \mathcal{H}$ ilb the universal family, and $D \subset \mathcal{H}$ ilb the discriminant divisor. Suppose $B \subset \mathcal{H}$ ilb is a smooth curve with corresponding family

$$
\mathcal{Y}:=\mathcal{U} \times{ }_{\mathcal{H} \text { ilb }} B \rightarrow B
$$

Then $B$ is transversal to each branch of the discriminant iff $\mathcal{Y}$ is regular and the fibers have ordinary double points.

Our transversality condition means that each branch of $D$ at $b$ is smooth and transverse to $B$.

Proof. Let $b \in B \cap D$ and $y \in Y_{b}$ a singularity corresponding to a branch $D^{\prime} \subset D$ at $b$. First suppose that $y$ is an isolated singularity of $\mathcal{Y}_{b}$. Then we have the formula $[22,2.8 .3]$

$$
\operatorname{mult}_{b}\left(B \cap D^{\prime}\right)=\mu\left(\mathcal{Y}_{b}, y\right)+\mu(\mathcal{Y}, y)
$$

the sum of the corresponding Milnor numbers. Thus the multiplicity is one if $\mu\left(\mathcal{Y}_{b}, y\right)=1$ and $\mu(\mathcal{Y}, y)=0$, i.e., $y \in \mathcal{Y}$ is nonsingular and $y \in \mathcal{Y}_{b}$ is an ordinary double point.

Now suppose $y$ fails to be isolated. If $d=2$ then the quadratic form defining $\mathcal{Y}_{b}$ has rank $\leq n-1$; the discriminant is defined by the determinant of an $(n+1) \times$ $(n+1)$ symmetric matrix and thus is singular when the matrix has rank $<n$. Otherwise, let $\Sigma \subset \mathcal{Y}_{b}$ denote the irreducible component of the singular locus containing $y$. Choose generic $y^{\prime}, y^{\prime \prime} \in \Sigma, y^{\prime} \neq y^{\prime \prime}$, and consider the hypersurfaces singular at both $y^{\prime}$ and $y^{\prime \prime}$, which form a codimension-2(n+1) linear subspace $L \subset \mathcal{H} i l b=\mathbb{P}\left(\Gamma\left(\mathcal{O}_{\mathbb{P}^{n}}(d)\right)\right)$. The generic hypersurface singular at $y^{\prime}$ (or $\left.y^{\prime \prime}\right)$ is contained in $D^{\prime}$ thus $L$ is contained in the singular locus of $D^{\prime}$.

\section{Higher-Dimensional FAno hypersurfaces}

Here we work over an uncountable algebraically closed field $k$ of characteristic zero.

We are grateful to James $\mathrm{M}^{\mathrm{c}}$ Kernan for pointing out the following amplification of $[11,5.9]$ 
Proposition 24. Let $X$ be a projective rationally connected variety with isolated terminal local complete intersection singularities. Then $X^{s m}$ is strongly rationally connected.

Proof. Let $\rho: \tilde{X} \rightarrow X$ denote a resolution of singularities of $X$, such that $\rho^{-1}\left(X^{s m}\right) \rightarrow X^{s m}$ is an isomorphism and $\tilde{X} \backslash \rho^{-1}\left(X^{s m}\right)$ is a normal crossings divisor with components $E_{1}, \ldots, E_{m}$.

We first show that $X^{s m}$ is rationally connected. Suppose that $\left(x_{1}, x_{2}\right) \in$ $X^{s m} \times X^{s m}$ is general, in the sense that it lies in the complement of a countable union of proper subvarieties. (Here we are using the fact that the base field is uncountable.) Then any morphism

$$
h: \mathbb{P}^{1} \rightarrow \tilde{X}, h(0)=x_{1}, h(\infty)=x_{2}
$$

is necessarily very free (cf. $[15,3.11])$.

Choose a very free imbedding $\tilde{f}: \mathbb{P}^{1} \rightarrow \tilde{X}$ with $\tilde{f}(0)=x_{1}, \tilde{f}(\infty)=x_{2}$, and image meeting $\rho^{-1}\left(X^{s m}\right)$. The induced curve in $X$ is denoted $f=\rho \circ \tilde{f}: \mathbb{P}^{1} \rightarrow X$. We may assume that $f\left(\mathbb{P}^{1}\right)$ meets the singularities of $X$; otherwise there is nothing to prove. Consequently, $\tilde{f}\left(\mathbb{P}^{1}\right)$ meets at least one of the $E_{i}$.

We compare dimensions of deformation spaces

$$
\operatorname{Def}(\tilde{f}):=\operatorname{Hom}\left(\mathbb{P}^{1}, \tilde{X} ; 0 \rightarrow x_{1}, \infty \rightarrow x_{2}\right)
$$

and

$$
\operatorname{Def}(f):=\operatorname{Hom}\left(\mathbb{P}^{1}, X ; 0 \rightarrow x_{1}, \infty \rightarrow x_{2}\right) .
$$

The first space has dimension

$$
-\operatorname{deg} \tilde{f}^{*} K_{\tilde{X}}-2 \operatorname{dim}(X)
$$

at $\tilde{f}$. By Theorem 2.10 of [13], the second space has dimension at least

$$
-\operatorname{deg} f^{*} K_{X}-2 \operatorname{dim}(X)
$$

the discrepancy formula

$$
K_{\tilde{X}}=K_{X}+\sum_{i} b_{i} E_{i}, \quad b_{i}>0
$$

then guarantees

$$
\operatorname{dim} \operatorname{Def}(\tilde{f})<\operatorname{dim} \operatorname{Def}(f)
$$


Composition by $\rho$ gives a morphism of deformation spaces

$$
\begin{aligned}
\iota_{\rho}: \operatorname{Def}(\tilde{f}) & \rightarrow \operatorname{Def}(f) \\
\tilde{g} & \mapsto \rho \circ \tilde{g}
\end{aligned}
$$

which is not dominant by dimension considerations. Let $g_{t}: \mathbb{P}^{1} \rightarrow X$ be a one-parameter deformation of $f$ such that

$$
\lim _{t \rightarrow 0} g_{t}=f,
$$

and $g_{t}(0)=x_{1}, g_{t}(\infty)=x_{2}$ for each $t$. Assume that $g_{t}$ is generic on the maximaldimension irreducible component of $\operatorname{Def}(f)$ passing through $f$. For generic $t$, write

$$
\tilde{g}_{t}: \mathbb{P}^{1} \rightarrow \tilde{X}
$$

is the lift of $g_{t}$ to $\tilde{X}$, which is also very free.

We claim $g_{t}\left(\mathbb{P}^{1}\right) \subset X^{s m}$. If not then we could repeat the argument above, finding a component of $\operatorname{Def}\left(g_{t}\right)$ with dimension strictly larger than the dimension of $\operatorname{Def}\left(\tilde{g}_{t}\right)$, contradicting our assumption.

We now prove that $X^{s m}$ is strongly rationally connected, by exhibiting a free curve in $X^{s m}$ through each point $x_{1} \in X^{s m}$. As above, let $\tilde{f}: \mathbb{P}^{1} \rightarrow \tilde{X}$ denote a free curve with $\tilde{f}(0)=x_{1}$ and passing through a general point of $\tilde{X}$. Write $f=\rho \circ \tilde{f}$ and repeat our dimension analysis, applied to the deformation spaces

$$
\operatorname{Def}(\tilde{f}):=\operatorname{Hom}\left(\mathbb{P}^{1}, \tilde{X} ; 0 \rightarrow x_{1}\right)
$$

and

$$
\operatorname{Def}(f):=\operatorname{Hom}\left(\mathbb{P}^{1}, X ; 0 \rightarrow x_{1}\right) .
$$

If $f\left(\mathbb{P}^{1}\right)$ is not contained in $X^{s m}$ then

$$
\operatorname{dim} \operatorname{Def}(\tilde{f})<\operatorname{dim} \operatorname{Def}(f)
$$

and we can choose $g_{t}: \mathbb{P}^{1} \rightarrow X$ generic on the component of maximal dimension. The lift $\tilde{g}_{t}: \mathbb{P}^{1} \rightarrow \tilde{X}$ remains free because it passes through a general point of $\tilde{X}$. Thus if $g_{t}\left(\mathbb{P}^{1}\right)$ were not in $X^{s m}$ then we could exhibit a component of $\operatorname{Def}\left(g_{t}\right)$ with dimension strictly larger than the dimension of $\operatorname{Def}\left(\tilde{g}_{t}\right)$, a contradiction.

Remark 25. The classification of terminal singularities in dimension three [19] shows they are quotients of isolated complete intersection singularities by the action of $\mathbb{Z} / r \mathbb{Z}$, where $r$ is the index of the singularity. Thus the hypotheses of Proposition 24 are quite natural. 
With further technical hypotheses, the proof of Proposition 24 can be extended to $r>1$.

Example 26. Examples of three-dimensional terminal singularities of index one include ordinary threefold double points

$$
w^{2}=x^{2}+y^{2}+z^{2} .
$$

For a complete list see $[17,6.4]$.

Theorem 1 then gives

Corollary 27. Let $X$ be a smooth rationally connected variety over $F=k(B)$. Suppose $X$ admits a regular proper model $\pi: \mathcal{X} \rightarrow B$ whose singular fibers have isolated terminal complete-intersection singularities. Then weak approximation holds for $X$ away from $S=\bar{B} \backslash B$.

Applying Lemma 23 we obtain

Corollary 28. Let $\mathcal{H i l b}=\mathbb{P}\left(\Gamma\left(\mathcal{O}_{\mathbb{P}^{n}}(d)\right)\right) \simeq \mathbb{P}^{\left(\begin{array}{c}n+d \\ d\end{array}\right)-1}$ denote the Hilbert scheme of hypersurfaces of degree $d \leq n, n \geq 4, \mathcal{U} \rightarrow \mathcal{H}$ ilb the universal family, and $D \subset \mathcal{H i l b}$ the discriminant divisor. Let $B \subset \mathcal{H i l b}$ be a smooth curve transverse to each branch of $D$ and

$$
\mathcal{X}:=\mathcal{U} \times{ }_{\mathcal{H} \text { ilb }} B \rightarrow B
$$

the corresponding family. Then $X=\mathcal{U} \times \mathcal{H}_{\text {Hilb }} \operatorname{Spec}(F)$ satisfies weak approximation away from $S=\bar{B} \backslash B$.

\section{REFERENCES}

[1] M. Artin. Algebraic approximation of structures over complete local rings. Inst. Hautes Études Sci. Publ. Math., (36):23-58, 1969.

[2] F. Bogomolov and Y. Tschinkel. Rational curves and points on K3 surfaces. Amer. J. Math., 127(4):825-835, 2005.

[3] E. Brieskorn. Die Aufösung der rationalen Singularitäten holomorpher Abbildungen. Math. Ann., 178:255-270, 1968.

[4] E. Brieskorn. Singular elements of semi-simple algebraic groups. In Actes du Congrès International des Mathématiciens (Nice, 1970), Tome 2, pages 279-284. Gauthier-Villars, Paris, 1971.

[5] J. L. Colliot-Thélène and P. Gille. Remarques sur l'approximation faible sur un corps de fonctions d'une variable. In Arithmetic of higher-dimensional algebraic varieties (Palo Alto, CA, 2002), volume 226 of Progr. Math., pages 121-134. Birkhäuser Boston, Boston, MA, 2004. 
[6] A. J. de Jong and J. Starr. Low degree complete intersections are rationally simply connected, 2006. preprint.

[7] O. Debarre. Variétés rationnellement connexes (d'après T. Graber, J. Harris, J. Starr et A. J. de Jong). Astérisque, 290:exposé 905, ix, 243-266, 2003. Séminaire Bourbaki. Vol. $2001 / 2002$.

[8] T. Graber, J. Harris, and J. Starr. Families of rationally connected varieties. J. Amer. Math. Soc., 16(1):57-67, 2003.

[9] J. Harris. Algebraic geometry, volume 133 of Graduate Texts in Mathematics. SpringerVerlag, New York, 1995.

[10] B. Hassett and Y. Tschinkel. Weak approximation over function fields. Inventiones mathematicae, 163:171-190, 2006.

[11] S. Keel and J. McKernan. Rational curves on quasi-projective surfaces. Mem. Amer. Math. Soc., 140(669):viii+153, 1999.

[12] A. Knecht. Approximation at Places of Bad Reduction for Degree Two del Pezzo Surfaces. PhD thesis, Rice University, Houston, Texas, 2007.

[13] J. Kollár. Cone theorems and bug-eyed covers. J. Algebraic Geom., 1(2):293-323, 1992.

[14] J. Kollár. Shafarevich maps and automorphic forms. M. B. Porter Lectures. Princeton University Press, Princeton, NJ, 1995.

[15] J. Kollár. Rational curves on algebraic varieties, volume 32 of Ergebnisse der Math. Springer-Verlag, Berlin, 1996.

[16] J. Kollár, Y. Miyaoka, and S. Mori. Rationally connected varieties. J. Algebraic Geom., 1(3):429-448, 1992.

[17] J. Kollár and N. I. Shepherd-Barron. Threefolds and deformations of surface singularities. Invent. Math., 91(2):299-338, 1988.

[18] D. Madore. Approximation faible aux places de bonne réduction sur les surfaces cubiques sur les corps des fonctions, 2004. preprint, to appear.

[19] S. Mori. On 3-dimensional terminal singularities. Nagoya Math. J., 98:43-66, 1985.

[20] M. Nagata. A generalization of the imbedding problem of an abstract variety in a complete variety. J. Math. Kyoto Univ., 3:89-102, 1963.

[21] P. Swinnerton-Dyer. Weak approximation and $R$-equivalence on cubic surfaces. In Rational points on algebraic varieties, volume 199 of Progr. Math., pages 357-404. Birkhäuser, Basel, 2001.

[22] B. Teissier. Introduction to equisingularity problems. In Algebraic geometry (Proc. Sympos. Pure Math., Vol. 29, Humboldt State Univ., Arcata, Calif., 1974), pages 593-632. Amer. Math. Soc., Providence, R.I., 1975.

[23] D.-Q. Zhang. Algebraic surfaces with nef and big anti-canonical divisor. Math. Proc. Cambridge Philos. Soc., 117(1):161-163, 1995.

Brendan Hassett

Department of Mathematics

Rice University, MS 136, Houston, Texas 77251-1892, USA 
E-mail: hassett@rice.edu

\section{Yuri Tschinkel}

Mathematisches Institut, Bunsenstr. 3-5, 37073 Göttingen, Germany Courant Institute, NYU, 251 Mercer str., New York, NY 10012, USA

E-mail: tschinkel@cims.nyu.edu 